\title{
Prevalence of vincristine induced-peripheral neuropathy among Sudanese cancer patients
}

\author{
Ethar Abdelmageed Imam ' , Alnada Ibrahim², Subish Palaian ${ }^{3}$, Mohamed Izham Mohamed Ibrahim ${ }^{4 *}$ \\ Department of Pharmacy, Clinical Pharmacist, Security Forces Hospital, Makkah, SAUDI ARABIA. \\ 2Department of Academic Affairs, Faculty of Pharmacy, University of Khartoum, Khartoum, SUDAN. \\ ${ }^{3}$ Department of Pharmacy Practice, College of Pharmacy, Gulf Medical University, Ajman, UNITED ARAB EMIRATES. \\ ${ }^{4}$ Clinical Pharmacy and Practice Section, College of Pharmacy, Qatar University, Al Tarfa, P.O. Box 2713, Doha, QATAR.
}

\begin{abstract}
Background: Increasing clinical evidence suggests treatment of cancer with neurotoxic agents results in some degree of peripheral neuropathy. Objective: The main objective of the study was to evaluate vincristine induced peripheral neuropathy among Sudanese cancer patients. Methods: A descriptive cross-sectional study was carried out at Radiation and Isotopes Center, Khartoum State, Sudan. All patients suffering from cancer with an age of 18 to 65 years were reviewed. A self-developed data collection form was used to collect the demographic data; drug use history; medical diseases; and vincristine cycles. The World Health Organization criteria were used to grade the side-effects. Data was collected for a period of two months and were analyzed descriptively. Results: Out of 78 patients reviewed, majority were females $(n=47,60.3 \%)$. Most of the patients were in the age group of $18-30(n=27,34.6 \%)$ and $51-60(n=27,34.6 \%)$ years. Twenty six $(33.3 \%)$ of the patients took analgesic and $15.3 \% \quad(n=12)$ experienced hypertension. Most of the patients went through more than 6 cycles of therapy with vincristine $(n=24,30.9 \%)$. Our study showed that 'altered bowel function (i.e constipation)' was the most important neuropathic change, which occurred in $62(79.5 \%)$ of the patients who received vincristine. The second common neuropathic change was the 'lack of ability
\end{abstract}

to go upstairs un-aided', which occurred in $37(47.4 \%)$ of patients receiving vincristine. Majority of the patients ( $n=37,47.5 \%)$ showed mild paresthesia as graded by the 'WHO grading scale'. Conclusion: More than three-fourth of the cancer patients experienced vincristine induced peripheral neuropathy. Healthcare professionals need some understanding regarding medical management that may decrease occurrence of neuropathy and a better planning and implementing special care for patients at risk.

Key words: Cancer, Chemotherapy, Peripheral Neuropathy, Sudan, Vincristine.

Correspondence :

Mohamed Izham Mohamed Ibrahim,

Professor of Social \& Administrative Pharmacy, Clinical Pharmacy and Practice Section, College of Pharmacy, Qatar University, Al Tarfa, P.O. Box 2713 Doha, QATAR.

Tel: (+974) 44035580 (GMT +3hrs)

Fax: (+974) 44035551

E-mail: mohamedizham@qu.edu.qa

DOI: 10.5530/jyp.2016.3.12

\section{INTRODUCTION}

Sudan, one of the largest African country (718,723 square miles) is experiencing a growing cancer problem. ${ }^{1}$ Although, the exact cancer burden and prevalence in Sudan is not known, it is reported that cancer cases have increased from a total of 303 cases in 1967 to 6303 cases in $2010 .^{2}$ The burgeoning cancer epidemic in Sudan carries many challenges that are characteristic of developing countries. ${ }^{3}$ It is reported that cancer was the third leading cause of death (accounting 5\% of total death) after malaria and viral pneumonia. ${ }^{4}$ The National Cancer Registry in Khartoum (the capital city of Sudan) was established in 2009. The country has only two cancer centers, located in Khartoum and Wad Madani, with capabilities to diagnose and treat cancer and the country has a population of approximately 38 million. These two centers receive referral patients from the entire country. It was reported that around 6771 new cancer cases were registered between the period 2009-2010; 3646 (53.8\%) cases were in women and $3125(46.2 \%)$ were in men. The most commonly type of cancer among female was breast cancer followed by leukemia, cervix and ovary, and in males prostate cancer was the most common followed by leukemia, lymphoma, oral, colorectal and liver and in children ( $<15$ yrs), leukemia was the most common cancer followed lymphoma. ${ }^{1}$

'Vincristine, belonging to the class Vinca alkaloids, works by inhibiting the cancer cells from separating into two new cells and thus blocking the growth of the cancer. ${ }^{5}$ It is widely used chemotherapy drug to treat various types of cancers and is commonly used in Sudan. Like any other cancer chemotherapeutic agents, vincristine also causes serious side effects. ${ }^{6}$ In addition, this drug should only be administered intravenously and never by any other route. In the past, there are reports vincristine has accidentally been administered via a spinal route, which leads to death in almost every case, thus making this a special drug for monitoring. ${ }^{7}$

The toxicity profile of the different anti tubulin drugs (class which vincristine belongs) varies in terms of their clinical presentation, incidence, severity, and outcomes, and the most neurotoxic being vincristine. ${ }^{8}$ Vincristine produces a predictable mixed motor and sensory neuropathy, as well as an autonomic neuropathy. Sensory symptoms are more severe than signs and start with numbness and tingling in a stocking distribution, glove distribution, or both. Peripheral neuropathy is the derangement in structure and function of peripheral motor, sensory and autonomic neurons. Vincristine induced neuropathy more commonly affects the distal portions of both hands and feet. ${ }^{9}$ One study reported the development of moderately severe peripheral neuropathy in 2 of 8 patients being treated with vincristine ( 1 to $4 \mathrm{mg}$ every 1 to 4 weeks, intravenously) for multiple myeloma in stable partial remission. It was discontinued in the 2 patients who developed peripheral neuropathy. ${ }^{10}$ These could cause peripheral neuropathic symptoms and signs. It is recommended for peripheral neurotoxic assessment during and after discontinuation of chemotherapy to monitor and study the occurrence of side effects. ${ }^{11}$ One recent study evaluated the genetic involvement of vincristine induced peripheral neuropathy and found an inherited polymorphism in the promoter region of CEP72 that was associated with increased risk and severity of vincristine-related peripheral neuropathy. ${ }^{12}$

In 2014, the American Society of Clinical Oncology (ASCO) performed a systematic review of relevant evidence to develop guidelines for prevention and treatment of chemotherapy-induced peripheral neuropathy (CIPN). Forty-eight randomized controlled trials were identified. This 
report summarizes the guidelines presented by ASCO. ${ }^{11}$ The induction of peripheral neuropathy is a common factor in limiting therapy with chemotherapeutic drugs. Little is known about the development of neuropathy due to vincristine among Sudanese cancer patients. Even though the use of vincristine is high in Sudan, it seems that information regarding vincristine peripheral neuropathy among Sudanese cancer patients is scarce in the literature. Vincristine seems to remain one of the most important drugs in the treatment of cancer in Sudan. This preliminary study explored the extent of cases and provided useful information to healthcare providers in the oncology areas and the aim of this study was to evaluate vincristine peripheral neuropathy among Sudanese cancer patients.

\section{METERIALS AND METHODS}

Study design: A descriptive cross sectional hospital-based study design was used in this study. It was carried out at Khartoum State, Sudan.

Study duration: During the period 10 December 2013 to 10 February 2014. The ethics committee at Radiation and Isotopes Center, Khartoum, Sudan approved the study.

Population: All patients suffering from cancer with aged 18 to 65 years were reviewed. Patients were excluded if family members have had a history of peripheral neuropathy.

Study setting: The study was carried out at the Radiation and Isotopes Center Khartoum. This is a Ministry of Health center for diagnosis and management of cancer disease in Sudan. It is one among the two cancer centers in the country. It is a 100-bed health facility. The other center in Wad Madani (that serves for $10 \%$ of the total Sudanese population) ${ }^{13}$ is excluded from the study due to feasibility reasons and unavailability of data.

Data collection tools: Data collection form collected the following patient's information: demographic data: age, gender, socioeconomic status; drug use history: analgesics, steroids; medical diseases history: diabetes mellitus, hypertension; and vincristine cycles. The WHO criteria (subjective measure) were developed at the initiative of the World Health Organization. ${ }^{14}$ Recommendations were described for standard- ized approaches to the recording of baseline data relating to the-patient, the tumor, laboratory and radiological data, the reporting of treatmentrelated toxicity and tumor response. The peripheral neuropathy section includes paresthesias, reflex decrease, and extent of motor loss as parameters. Data was analyzed descriptively as frequency and percentages using SPSS Version 22.

\section{RESULTS}

Demographic profile of the patients: Out of 78 patients reviewed, majority of the cases were females $(n=47,60.3 \%)$. Most of the patients were in the age group of $18-30(n=27,34.6 \%)$ and $51-60(n=27,34.6 \%) .33 .3 \%$ of the patients took analgesic and $15.3 \%$ experienced hypertension (Table 1).

Disease background and therapy: Most of the patients went through more than 6 cycles of therapy with vincristine $(\mathrm{n}=24,30.9 \%)$ (Table 2).

Vincristine induced peripheral neuropathy: Our study showed that altered bowel function (constipation) was the most important neuropathy change, which occurred in $62(79.5 \%)$ of the Sudanese cancer patients who received vincristine. The second common neuropathic change was the lack of ability to go upstairs un-aided (a parameter used to evaluate peripheral neuropathy), which occurred in 37 (47.4\%) of patients receiving vincristine (Table 3 ).

In this study, almost half of our patients ( $\mathrm{n}=37 ; 47.5 \%)$ showed mild paresthesia according to WHO scale (Figure 1).

\section{DISCUSSION}

Although, chemotherapy has resulted in substantial positive outcomes for cancer patients, it has caused substantial toxicity to the cancer patients. Our study found a high percentage of patients experienced neuropathy due to vincristine. The side effect such as peripheral neuropathy could cause discontinuation of therapy and negatively affect the patient's quality of life. ${ }^{15}$ Our findings indicated that most of the cancer patients experienced non-normal bowel function after taking vincristine, problem of getting upstairs unaided and problem walking unaided. The incidence of side effects ranged from $12.8-79.5 \%$ which was higher than documented by several studies (30-47\%). ${ }^{15}$ One Indian study found a

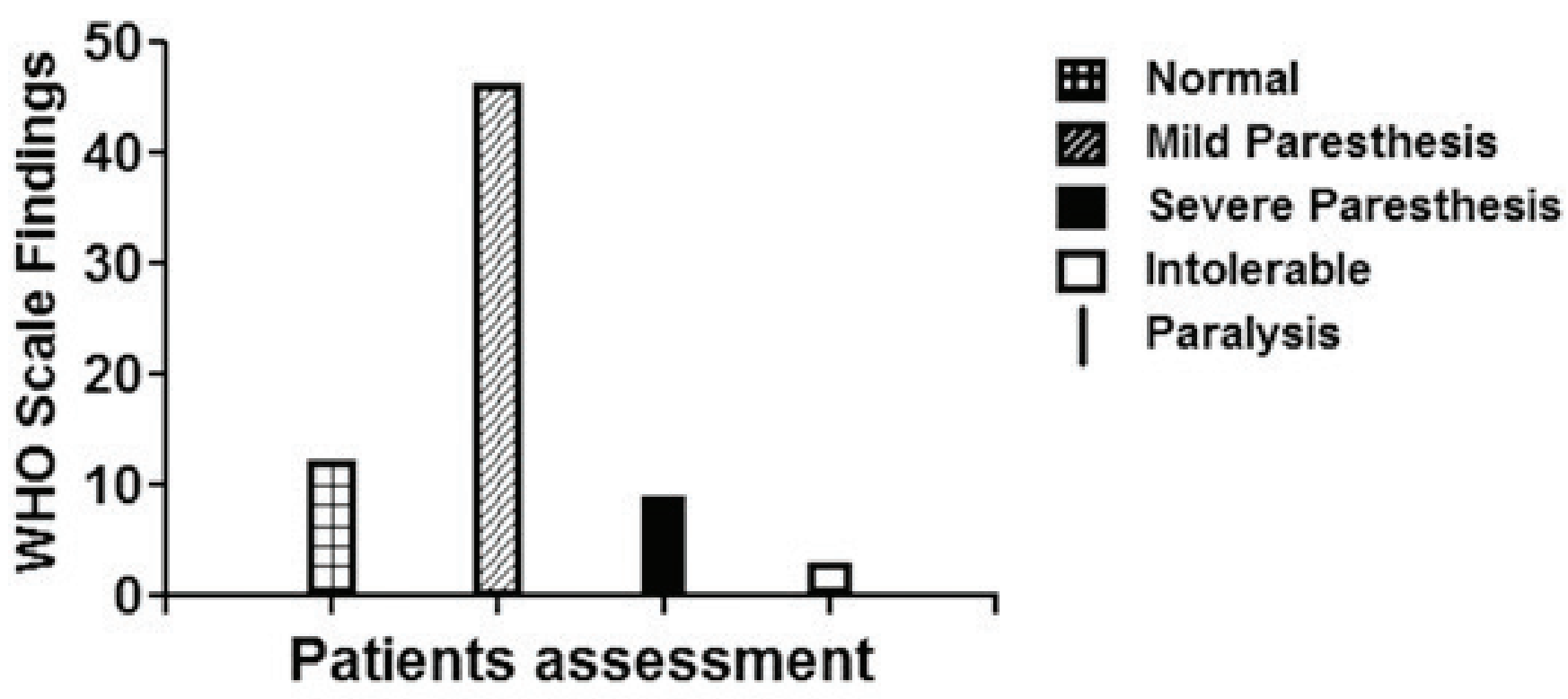

Figure 1: WHO grading scales for peripheral neuropathy. 
IMAM et al.: Prevalence of vincristine induced-peripheral neuropathy

Table 1: Demographic features, drug and medical information of cancer patients

\begin{tabular}{ccc}
\hline No & Parameters & Number (\%) \\
\hline 1. & Total number of the patients studied & $78(100)$ \\
2. & Gender & $31(39.7)$ \\
\multicolumn{3}{c}{ (a) Male } \\
3. & (b) Female & $47(60.3)$ \\
(a) & Age Group (Years) \\
(b) & $18-30$ & $27(34.6)$ \\
(c) & $31-40$ & $10(12.8)$ \\
(d) & $41-50$ & $09(11.6)$ \\
(e) & $51-60$ & $27(34.6)$ \\
4. & $>60$ & $05(6.4)$ \\
(a) & Drug History & \\
(b) & Analgesics & $26(33.3)$ \\
(c) & Steroids & $09(11.5)$ \\
5. & Other drugs & $43(55.2)$ \\
(a) & Medical Diseases & $12(15.3)$ \\
(b) & Hypertension & $04(5.1)$ \\
(c) & Diabetes Mellitus & $62(79.6)$ \\
\hline & Other diseases & \\
\hline
\end{tabular}

\begin{tabular}{|c|c|}
\hline Vincristine Cycles & Number (\%) \\
\hline One cycle & $12(15.4)$ \\
\hline Two cycles & $12(15.4)$ \\
\hline Three cycles & $09(11.5)$ \\
\hline Four cycles & $03(3.8)$ \\
\hline Five cycles & $09(11.5)$ \\
\hline Six cycles & $09(11.5)$ \\
\hline$>$ Six cycles & $24(30.9)$ \\
\hline
\end{tabular}

Table 3: Vincristine induced peripheral neuropathy in cancer patients

\begin{tabular}{cc}
\hline Patients findings & Number (\%) \\
Yes & $68(87.2)$ \\
No & $10(12.8)$ \\
Walk Unaided & \\
Yes & $50(64.1)$ \\
No & $28(35.9)$ \\
Go Upstairs Unaided & \\
Yes & $41(52.6)$ \\
No & $37(47.4)$ \\
Temperature Sense & $68(87.2)$ \\
Yes & $10(12.8)$ \\
No & \\
Bowel Function BEFORE Vincristine Therapy & $75(96.2)$ \\
Normal & $03(3.8)$ \\
Not Normal & $16(20.5)$ \\
Bowel Function AFTER Vincristine Therapy & $62(79.5)$ \\
\hline Normal &
\end{tabular}


relatively higher incidence of vincristine-induced neuropathy in Indian children and authors attributed it to coexistence of severe malnutrition in those patients. ${ }^{16}$

According to one research finding, vincristine causes peripheral neuropathy and the patients who suffers peripheral neuropathy experiences problems related to cardiovascular, gastrointestinal and genitourinary systems. ${ }^{16}$ In addition, patients who experienced peripheral neuropathy, a side-effect from chemotherapy could be disabled after using antineoplastic agents. ${ }^{17}$ One study noticed, in children the lower age could resulted in greater sensitivity of some nerves to vincristine and thus showing a motor predominant neuropathy in children. ${ }^{18}$ Patients experiencing this side effects would require reduction or cessation of the chemotherapy. ${ }^{11,19,20}$ Worst case that could happen is increase in cancerrelated morbidity and mortality. The frequency and severity of the sideeffects depend very much on the dose, duration and schedule of the chemotherapy used. ${ }^{15}$

It is recommended for performing routine neuropathic pain analysis on patients following various investigations. ${ }^{21}$ One study provided evidence for overcoming this effect. Authors strongly recommended that intravenous calcium and magnesium therapy can lessen the development of oxaliplatin-induced peripheral neuropathy. Vitamin E may also reduce the development of this effect. Other agents that could be used but need more strong evidence are glutamine, glutathione, $\mathrm{N}$-acetylcysteine, oxcarbazepine, and xaliproden. ${ }^{20}$

In clinical practice settings, peripheral neuropathy is normally screened in patients treated with chemotherapy drugs like vincristine. ${ }^{22}$ There are several chemotoxicities grading systems i.e. combination of subjective (patient questionnaire) and objective (clinical evaluation) parameters, that could be used for various levels of paresthesia. One of the widely used grading systems is the WHO criteria. ${ }^{14}$ Our finding using the WHO criteria indicated that close to half of the patients experienced mild paresthesia. Vincristine-induced peripheral neuropathy is dose dependent, as up to $60 \%$ of patients may develop a clinically significant (grade 1-2) primarily sensory or sensorimotor neuropathy at vincristine cumulative doses between $30-50 \mathrm{mg} \cdot{ }^{21}$ This finding indicates that vincristine was well tolerated by nearly half of the Sudanese cancer patients. This finding is comparable to a study from Britain. ${ }^{23}$ However, the induction and maintenance of vincristine in the British study was measured by objective evaluation methods, while in our study we used a subjective questionnaire evaluation method. This point suggests the need for future prospective studies on vincristine peripheral neuropathy in Sudan. Quantitative assessment is a must on patients receiving chemotherapy and it should be continued even after patients have stopped taking the drug.

\section{Clinical implications of the study}

Understanding the incidence of disabling peripheral neuropathy is important because it has a significant negative impact on patient's quality of life. Oncologists and other healthcare providers must realize the sideeffect experienced by cancer patients receiving chemotherapy. They need some understanding regarding medical management that may decrease neuropathy and planning and implementing special care for patients at risk. Assessment is important during treatment and even after discontinuation of treatment.

\section{Study limitations}

The study is only preliminary and looked at limited number of samples and the findings could not be generalized. This is a descriptive study and we are not able to make any conclusive remarks. Around $43 \%$ of the patients could not recalled the drugs taken in the past, while in $62 \%$ of the patients, other diseases experienced by them could not be identified from the medical record. These two factors could be related to peripheral neuropathy, but could not be confirmed. Moreover, the cancer center in Wad Madani (one among the two cancer centers in the country) was not studied in this research and hence cannot generalize this findings to the entire country.

\section{Study recommendations}

A nationwide study should be conducted to better understand the extent of vincristine and other chemotherapy-induced neuropathy among cancer patients in the country. The future study should also analyze the association of neuropathy and cumulative dose and type of chemotherapy received by patients.

\section{CONCLUSION}

In conclusion, most of the cancer patients experienced vincristine peripheral neuropathy especially affecting the bowel function and going up the stairs. Almost half of our patients showed mild paresthesia according to WHO scale. Healthcare professionals need some understanding regarding medical management that may decrease neuropathy, and planning and implementing special care for patients at risk.

\section{ACKNOWLEDGEMENT}

Our special thanks go to all the staff of the National Radiation and Isotopes Center, Khartoum, Ministry of Health, Sudan for their cooperation and assistance.

\section{CONFLICT OF INTEREST}

The author declare no conflict of interest.

\section{ABBREVIATIONS USED}

ASCO: American Society of Clinical Oncology; CIPN: Chemotherapy-induced peripheral neuropathy; WHO: World Health Organization.

\section{ABOUT AUTHORS}

Ethar Abdelmageed Imam: Obtained her MSc degree in Clinical Pharmacy from Khartoum University in 2014. The thesis was on vincristine peripheral neuropathy in Sudanese cancer patients. Currently, she is practicing as a clinical pharmacist at the Security Forces Hospital in Saudi Arabia.

Alnada A. M. Ibrahim: Is the Deputy Dean for Academic Affairs, and Assistant Professor at the Department of Pharmaceutics, Faculty of Pharmacy, University of Khartoum, Khartoum, Sudan.

Subish Palaian is an Associate Professor of Pharmacy Practice at College of Pharmacy, Gulf Medical University, Ajman United Arab Emirates. He obtained his PhD from UniversitiSains Malaysia, Penang, Malaysia in the year 2010. His major research interestsinclude pharmacovigilance, drug promotion, patient counseling, drug information, drug utilization evaluation and teaching safe use of medicines to health professionals and healthcare related students. 
Mohamed Izham Mohamed Ibrahim Is a Professor of Social and Administrative Pharmacy at College of Pharmacy, Qatar University, Doha, Qatar. He graduated with a bachelor degree in Pharmacy from UniversitiSains Malaysia in 1990 and obtained PhD degree in Pharmacy Administration from the Philadelphia College of Pharmacy \& Science (PCPS), Philadelphia, USA in 1995 with a cognate in Pharmaceutical Policy \& Supply Management, Pharmacoeconomics, Pharmacoepidemiology, Socio-behavioral Aspects of Pharmacy, and Pharmacy Management \& Marketing.

\section{REFERENCES}

1. Saeed IE, Weng HY, Mohamed KH, Mohammed SI. Cancer incidence in Khartoum, Sudan: First results from the cancer registry, 2009-2010. Cancer Med. 2014;3(4):1075-84.

2. Elamin A, Ibrahim ME, Abuidris D, Mohamed KEH, Mohammed SI. Part I: Cancer in Sudan-burden, distribution, and trends breast, gynecological, and prostate cancers. Cancer Med. 2015;4(3):447-56

3. Hamad HMA. Cancer initiatives in Sudan. Ann Oncol. 2006;17(Supplement 8): viii32-36

4. Saeed IE, Abuidris DA, Mohamed KEH, Mohammed SI. Cancer registry in Sudan: A brief overview. Internet J Epidemiol. 2013;11(2):1-5. [cited March 24, 2016]. Available from: http://ispub.com/lJE/11/2/14574

5. Cancer Research UK. Vincristine. [cited January 26, 2016]. Available from: http:// www.cancerresearchuk.org/about-cancer/cancers-in-general/treatment/cancerdrugs/vincristine

6. Cancer.Net. Side effects. [cited January 26, 2016]. Available from: http://www. cancer.net/navigating-cancer-care/side-effects

7. Patient safety workshop. Learning from error. World Health Organization, 2008. [cited January 26, 2016]. Available from: http://www.who.int/patientsafety/ activities/technical/vincristine_learning-from-error.pdf

8. Grisold W, Cavaletti G, Windebank AJ. Peripheral neuropathies from chemotherapeutics and targeted agents: Diagnosis, treatment, and prevention. Neuro Oncol. 2012;14(Suppl 4):iv45-54.

9. DeAngelis LM, Gnecco C, Taylor L and Warrell RP Jr. Evolution of neuropathy and myopathy during intensive vincristine/corticosteroid chemotherapy for nonHodgkin's lymphoma. Cancer. 1991;67(9):2241-6.

10. Alberts DS, Durie BG, Salmon SE. Treatment of multiple myeloma in remission with anticancer drugs having cell cycle specific characteristics. Cancer Treat Rep. 1977;61(3):381-8

11. Hershman DL, Lacchetti C, Dworkin RH, Lavoie Smith EM, Bleeker J, Cavaletti G. Prevention and management of chemotherapy-induced peripheral neuropathy in survivors of adult cancers: American Society of Clinical Oncology Clinical
Practice Guideline. J Clin Oncol. 2014;32(18):1941-67.

12. Berg SL, Parsons DW. The pharmacogenomics of vincristine-induced neuropathy: On pins and needles. JAMA Oncol. 2015;1(7):975-6.

13. Awadelkarim KD, Mariani-Costantini R, Elwali NE. Cancer in the Sudan: an overview of the current status of knowledge on tumor patterns and risk factors. Sci Total Environ. 2012;423(15):214-28.

14. Miller AB, Hoogstraten $B$, Staquet $M$, Winkler $A$. Reporting results of cancer treatment. Cancer. 1981;47(1):207-14.

15. Stubblefield MD, Burstein HJ, Burton AW, Custodio CM, Deng GE, Ho M NCCN Task Force Report: Management of Neuropathy in Cancer. J Natl Compr Canc Netw. 2009;7(Suppl 5):S1-S26; quiz S27-8.

16. Gomber $S$, Dewan $P$, Chhonker D. Vincristine induced neurotoxicity in cancer patients. Indian J Pediatr. 2010;77(1):97-100

17. Seretny M, Currie GL, Sena ES, Ramnarine S, Grant R, MacLeod MR. Incidence, prevalence, and predictors of chemotherapy-induced peripheral neuropathy: A systematic review and meta-analysis. Pain. 2014;155(12):2461-70.

18. Courtemanche $H$, Magot A, Ollivier $Y$, Rialland F, Leclair-Visonneau L, Fayet $G$. Vincristine-induced neuropathy: Atypical electrophysiological patterns in children. Muscle Nerve. 2015;52(6):981-5.

19. Cavaletti G, Marmiroli P. Chemotherapy-induced peripheral neurotoxicity. Nat Rev Neurol. 2010;6(12):657-66.

20. Wolf S, Barton D, Kottschade L, Grothey A, Loprinzi C. Chemotherapy-induced peripheral neuropathy: Prevention and treatment strategies. Eur $\mathrm{J}$ Cancer. 2008;44(11):1507-15.

21. Kraychete DC, Sakata RK. Painful peripheral neuropathies. Rev Bras Anestesiol. 2011;61(5):641-58.

22. PostmaTJ, Heimans JJ. Grading of chemotherapy-induced peripheral neuropathy. Ann Oncol. 2000;11(5):509-13.

23. MacLennan ICM, Cusick J. Objective evaluation of the role of vincristine in induction and maintenance therapy for myelomatosis. Br J Cancer. 1985;52(2):153-8. 